\title{
On the Random Access Procedure of NB-IoT Non-Terrestrial Networks
}

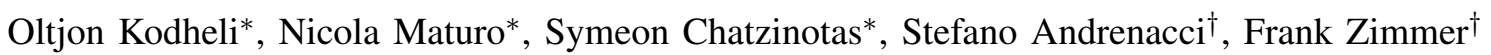 \\ ${ }^{*}$ SnT - Interdisciplinary Centre for Security, Reliability and Trust, University of Luxembourg, Luxembourg \\ Email: \{oltjon.kodheli, nicola.maturo, symeon.chatzinotaz\}@uni.lu \\ ${ }^{\dagger}$ SES S.A., Betzdorf, Luxembourg \\ Email: \{stefano.andrenacci, frank.zimmer\}@ses.com
}

\begin{abstract}
The standardization of the $5 \mathrm{G}$ systems has recently entered in an advanced phase, where non-terrestrial networks will be a new key feature in the upcoming releases. Narrowband Internet of Things (NB-IoT) is one of the technologies that will address the massive machine type communication (mMTC) traffic of the 5G. To meet the demanding need for global connectivity, satellite communications can provide an essential support to complement and extend the NB-IoT terrestrial infrastructure. However, the presence of the satellite channel comes up with new demands for the NB-IoT procedures. In this paper, we investigate the main challenges introduced by the satellite channel in the NB-IoT random access procedure, while pointing out valuable solutions and research directions to overcome those challenges.
\end{abstract}

\section{INTRODUCTION}

NB-IoT is one of the most appealing low-power widearea networks (LPWAN) technologies, expected to play an important role in the fifth generation mobile communication $(5 \mathrm{G})$ network. In order to guarantee a worldwide coverage to the low-cost IoT devices distributed all over the globe, satellite connectivity is a key asset due to their large footprint on Earth. Their role is irreplaceable, especially in remote areas where the terrestrial network is impossible to reach, or the investment towards it cannot be justified.

As a matter of fact, after two years of a study phase [1], it is now approved by the 3rd Generation Partnership Project (3GPP) that non-terrestrial network (including satellites) will be a new key feature of the $5 \mathrm{G}$, and a work item (WI) has already started for Release 16 and 17 [2]. Furthermore, it is agreed that initial studies on the support of NB-IoT by non-terrestrial networks (NTN) will be performed as well, potentially targeting Release 17 [3]. The study will focus on the adaptations of the NB-IoT protocol taking into account the satellite channel characteristics, which are very different compared to the terrestrial one. As an initial phase, only the low Earth orbit (LEO) and geostationary orbit (GEO) will be considered.

Several contributions in the literature have studied such systems, showing the fundamental features and the role of the satellites in the 5G IoT communications [4], [5]. The authors in [6] assess the impact of large delays and Doppler

This work was supported by the Luxembourg National Research Fund (FNR) under Industrial Fellowship Scheme with industrial partner SES S.A., project title "Communication algorithms for end-to-end satellite-IoT (SATIOT)", grant FNR12526592. shifts in two scenarios of interest for future 5G NTN systems, enhanced Mobile BroadBand (eMBB) services and NB-IoT. In [7] a resource allocation technique for a satellite-based NB-IoT system is proposed, which significantly reduces the differential Doppler shift experienced in a LEO satellite.

In parallel with the above-mentioned efforts, which try to adapt the already existing protocols so as to meet the satellite channel characteristics, other works in the literature focus on proposing new technologies. For example, the authors in [8] come up with a new air interface for NB-IoT based on Turbo-FSK modulation, able to deal with large Doppler effects and delays. Moreover, in [9] an NB-IoT receiver architecture is presented, that takes into consideration satellite channelspecific impairments. However, following this approach requires totally new chipsets for the NB-IoT, which goes against the 3GPP objectives for future releases.

One of the most important procedures worth analyzing for the NB-IoT NTN is the random access (RA) procedure, since it allows the NB-IoT user equipments (UEs) to initiate uplink data transmission, achieve uplink synchronization and obtain a permanent ID in the network. Please note that in general, the IoT applications are based on uplink data reports (e.g. monitoring applications), hence a failed RA procedure would make the whole NB-IoT system useless. To the best of our knowledge, there exists no work in the literature which specifically evaluate the RA procedure and all its steps of a satellite-based NB-IoT system. Other works targeting the RA for 5G NTN exist [10], however the technical peculiarities of the NB-IoT system are quite different and a new investigation is needed.

This paper aims to make a complete analysis of the NB-IoT NTN random access procedure. The main challenges imposed by the presence of the satellite channel, under different orbits, in each step of this essential procedure, will be examined. Last but not least, new adaptations on the RA procedure to overcome the satellite channel impairments will be proposed. The solutions that already exist in the literature for the $5 \mathrm{G}$ NTN scenarios will be also included, and their feasibility for the NB-IoT NTN case will be discussed.

\section{NB-IOT NTN REFERENCE SCENARIOS}

The identified architecture options of a non-terrestrial network providing access to NB-IoT user equipments are shown 


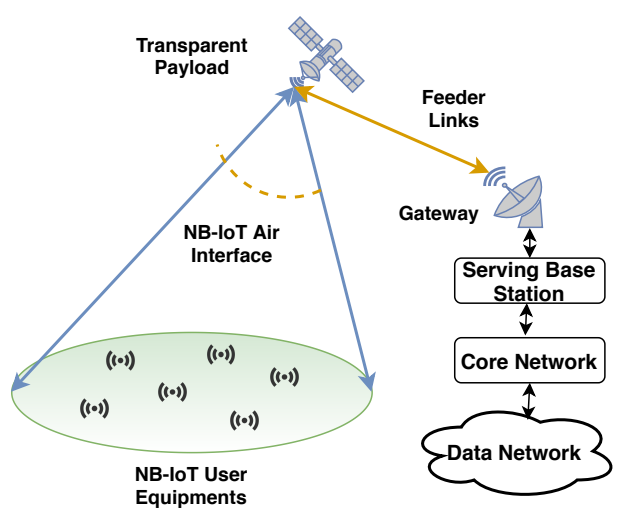

Fig. 1. NB-IoT NTN with transparent payload

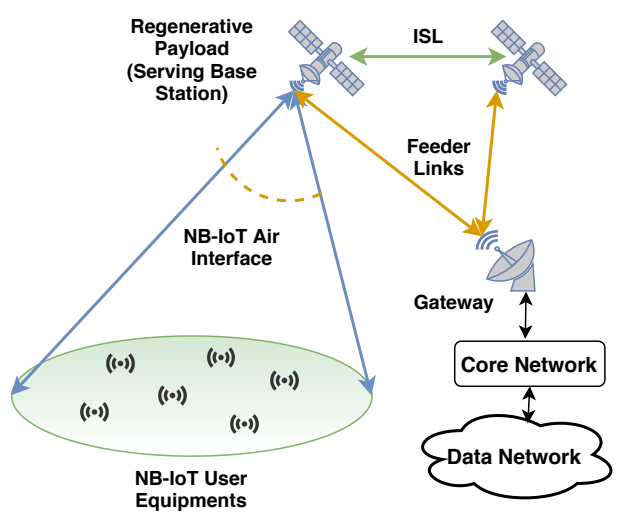

Fig. 2. NB-IoT NTN with regenerative payload

in Fig. 1 and Fig. 2. It is worth emphasizing here that these architecture options are already defined for the 5G NTN in 3 GPP [2]. Since it is already agreed that $5 \mathrm{G}$ will continue to address the mMTC traffic by further evolving NB-IoT and LTE-M [11], the same architecture options hold for the NB-IoT NTN as well. Basically, they differ from each-other depending on the payload, orbit, and cell type.

We consider two types of payloads, transparent and regenerative. In case of a transparent payload, the satellite acts as a relay, and provides the link between the NB-IoT users and the serving base station (BS), which are on ground. In contrast, in case of a regenerative payload, the BS functionalities can be performed at the satellite, and an inter-satellite link (ISL) can help for handover procedures.

Another important aspect is the type of cell that contains the NB-IoT UEs. Two types of cells have been identified, Earth-fixed cells and Earth-moving cells. In the former case (see Fig. 3), the cell is fixed and all the UEs inside the cell will have a certain coverage time by the satellite. To increase this coverage time, each satellite has the capability to steer beams towards fixed points on Earth. This can be realized through a mechanically steerable beam or a beamforming (BF) technique. On the other hand, an Earth-moving cell (see Fig. 4) will move with the same speed as the satellite. In such a case, the location of the users inside the cell will change

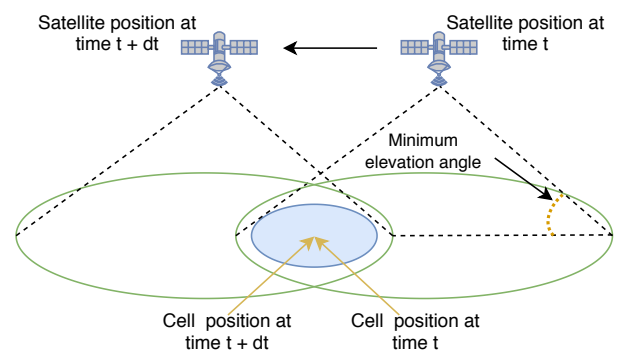

Fig. 3. Earth-fixed cells

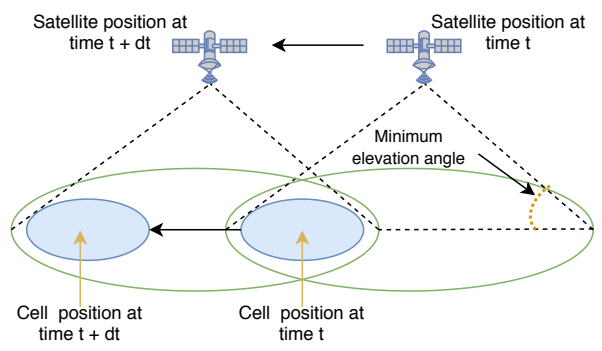

Fig. 4. Earth-moving cells

TABLE I

NB-IOT NTN SCENARIO OPTIONS

\begin{tabular}{|l|l|l|}
\hline & $\begin{array}{l}\text { Transparent } \\
\text { payload }\end{array}$ & $\begin{array}{l}\text { Regenerative } \\
\text { payload }\end{array}$ \\
\hline GEO based & Scenario A & Scenario B \\
\hline $\begin{array}{l}\text { LEO based with Earth- } \\
\text { fixed cells }\end{array}$ & Scenario C1 & Scenario D1 \\
\hline $\begin{array}{l}\text { LEO based with Earth- } \\
\text { moving cells }\end{array}$ & Scenario C2 & Scenario D2 \\
\hline
\end{tabular}

dynamically, and there will be constantly new users entering and going out of the cell. An advantage of this cell type is that it does not require a mechanical steering or BF, resulting in a lower satellite cost.

Regarding the orbit type, it is agreed in 3GPP that as a starting phase only the LEO and GEO satellite orbits will be considered [2], hence we will refer hereafter only to these orbits. As it is well-known, a GEO satellite has an altitude of $35,786 \mathrm{~km}$, whereas for the LEO two possible altitudes are defined, $600 \mathrm{~km}$ and $1200 \mathrm{~km}$. Please note that throughout the paper we will use only the $600 \mathrm{~km}$ altitude to make the analysis. Nevertheless, changing the altitude for the LEO satellite will not impact our analysis, but only certain parameters.

The possible scenario options built upon the previously stated considerations, are summarized in Table I, similar to the ones for $5 \mathrm{G}$ NTN.

\section{A BRIEF OVERVIEW OF THE NB-IOT RANDOM ACCESS PROCEDURE}

The RA procedure is a very crucial step of the NB-IoT network since it allows the NB-IoT UEs to initiate uplink data transmission, achieve uplink synchronization, and obtain 
a permanent ID. It follows the same steps as in LTE and will be detailed below. An illustration of the message exchange that occurs in NB-IoT RA procedure, is given in Fig. 5.

1) Message 1: After achieving downlink synchronization, the UEs will send a random access preamble to the serving $\mathrm{BS}$, by using the physical random access channel (NPRACH). This allows the BS to estimate the round trip delay (RTD) for each UE, based on the time of arrival (ToA) of the received NPRACH signal. Since NB-IoT employs a singlecarrier frequency division multiple access (SC-FDMA) for the uplink transmission, it is essential to align the received signals from multiple devices, both in time and frequency. The BS will use the ToA estimate, for determining a timing advance (TA) to be applied by each UE, for synchronizing their uplink transmissions. What is worth emphasizing here is that at this step the UEs will compete for the same NPRACH channel, hence packet collision may occur if two UEs randomly choose the same preamble configuration.

2) Message 2: If the BS detects an NPRACH preamble, it sends back a random access response (RAR), also known as Message 2. The RAR contains the TA parameter, which enables the time synchronization of the UEs. Besides, the RAR further encloses scheduling information pointing to the radio resources that the UEs has to use to transmit the subsequent messages. Basically, from this step on, the transmission of the data, either in downlink or uplink, is orchestrated by the BS. This means that the BS guarantees that different resources (time/frequency) are assigned to various UEs in order to avoid packet collision.

3) Message 3: In Message 3, the device will include its identity as well as a scheduling request. Furthermore, it will also report its data volume status and power headroom, to facilitate the base station scheduling and power allocation decision for subsequent transmissions. At this step, the hybrid automatic repeat request (HARQ) protocol is used by both, the UE and the BS, for the message exchange. Basically, after each packet transmission, the UE has to wait for an acknowledgment (ACK) or non-ACK (NACK) by the BS, or

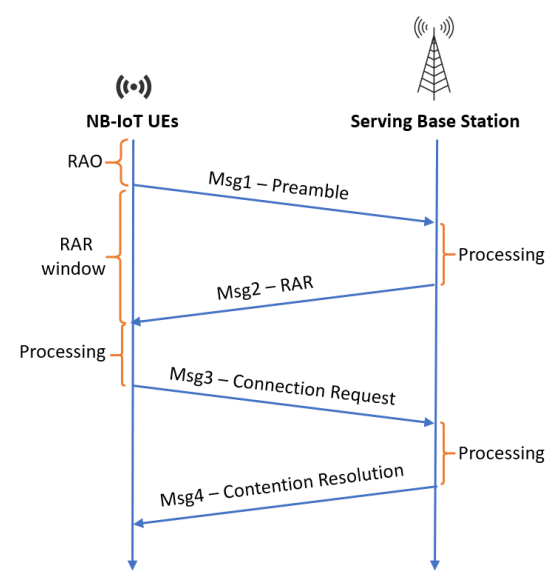

Fig. 5. Four message exchange that occurs in NB-IoT RA procedure

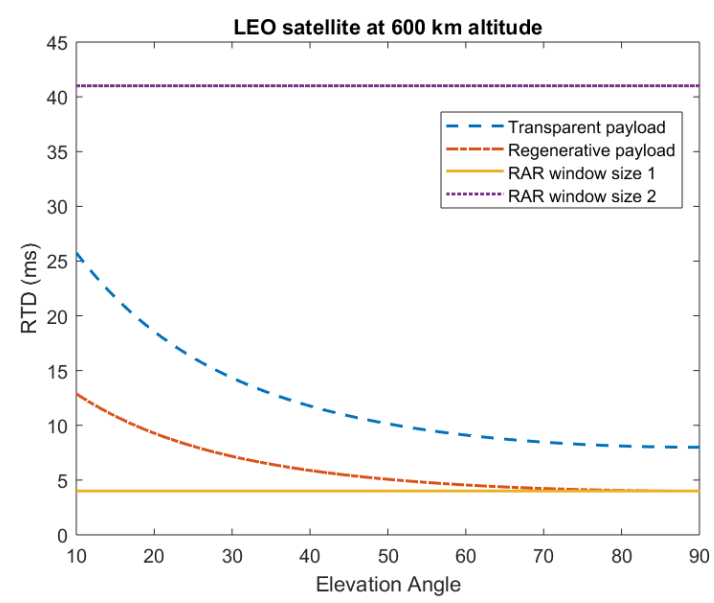

Fig. 6. RTD vs elevation angle

vice versa, to know whether the packet was correctly received. If not, the same packet is retransmitted.

4) Message 4: In Message 4, the network assigns a permanent ID to the UEs which had a successful RA procedure. At this point, the UEs make the transition from radio resource control (RRC) idle to RRC connected mode.

For the interested readers, more details on the RA procedure can be found in [12].

\section{The MAin CHALlenges of THE RA PROCEDURE OVER A NON-TERRESTRIAL NETWORK}

This section aims to investigate and describe the challenges that will be imposed by the satellite channel, on the RA procedure of an NB-IoT network.

\section{A. Round Trip Delay}

When the UEs send the preamble in message 1 to start the RA procedure, they wait for a limited amount of time to get the RAR message from the serving BS. In fact, in a terrestrial NB-IoT network, there exist two RAR window configurations, $4 \mathrm{~ms}$ and $41 \mathrm{~ms}$. The choice of the one or the other by the device, will depend on the signal to noise ratio (SNR) estimate of the downlink signal, and on the number of repetitions used to transmit the NPRACH preamble. For more details, please refer to Table 5.1.4-1 in [13].

It is worth highlighting here that the RAR window size should be large enough, in order to allow the preamble to be transmitted, processed at the BS and sent back to the device. The propagation time of the signal from the UE to the BS and vice versa is known as the round trip delay (RTD). While in a terrestrial case the RTD is very small $(<1 \mathrm{~ms})$, in a satellite scenario it becomes an influential factor to carefully consider. For a regenerative satellite, in which we assume the BS to be on-board, it can be calculated by the following formula:

$$
R T D_{\text {regen }}=2 \cdot D / c
$$

where $c$ is the speed of light, and $D$ is the distance from the UE to the satellite, known also as the slant range. In case of 
a transparent payload, by approximating the distance from the satellite to the $\mathrm{BS}$ on ground with the slant range $D$, then

$$
R T D_{\text {transp }} \approx 4 \cdot D / c
$$

Regarding the slant range, it is characterized as a function of the satellite parameters by the successive equation [1]:

$$
D=\sqrt{R_{E}^{2} \cdot \sin (\alpha)^{2}+h_{s}^{2}+2 \cdot R_{E} \cdot h_{s}}-R_{E} \cdot \sin (\alpha)
$$

where $R_{E}$ is the radius of Earth, $\alpha$ is the satellite elevation angle, and $h_{s}$ is the altitude of the satellite. Fig. 6 yields the range of RTD values for a LEO satellite under different elevation angles. For a GEO satellite, the $R T D_{\text {regen }} \approx 270$ $m s$ and $R T D_{\text {transp }} \approx 540 \mathrm{~ms}$. These values hold regardless of having an Earth-moving or an Earth-fixed cell, and will definitely have a significant impact on the RA procedure in two directions, as pointed out below.

1) RAR window size: As it can be noted by the results in Fig. 6, the RAR window size 1 is not big enough to account for the increased RTD in a satellite channel, even for the lowest satellite orbit and regenerative payload. In contrast, window size 2 will not impose a problem for such orbit specifications. However, for higher altitudes, for example in case of a GEO satellite, it is still insufficient to cover up the high RTD.

2) HARQ protocol: The HARQ protocol used in message 3 and message 4 data exchange, plays a role in increasing the link reliability of the communication. Nevertheless, it directly impacts the overall delay of the RA procedure, since it doubles the number of messages exchanged by the UEs and BS for the last two steps. Clearly, the higher the RTD, the slower the RA procedure would be. Comparing the RTD of a typical terrestrial NB-IoT network (lower than $1 \mathrm{~ms}$ ) with that over a GEO satellite, the rate in which the RA procedure slows down may be as high as 540. Alternative solutions should be considered.

\section{B. Differential RTD}

Before initiating the RA procedure, the UEs are not synchronized in time. Therefore, when many UEs attempt to access the network through message 1 transmission, their preambles will be misaligned because their distance from the BS is different. For the protocol to work, this timemisalignment should not overcome the cyclic prefix (CP) length. As a result, the differential RTD allowed among various UEs becomes a deciding factor for the radius of the cell. UEs outside this radius will fail to have a successful RA procedure. The relation between the CP length $\left(T_{c p}\right)$ and the NPRACH cell radius $\left(R_{c e l l}^{(R A)}\right)$ can be expressed by the following formula:

$$
R_{\text {cell }}^{(R A)}=c \cdot T_{c p} / 2
$$

This cell radius guarantees that the difference between the RTD of the closest user to the BS (the one in the center of the cell for a terrestrial network) and the furthest one (the one in the edge), does not exceed the CP length. It directly depends

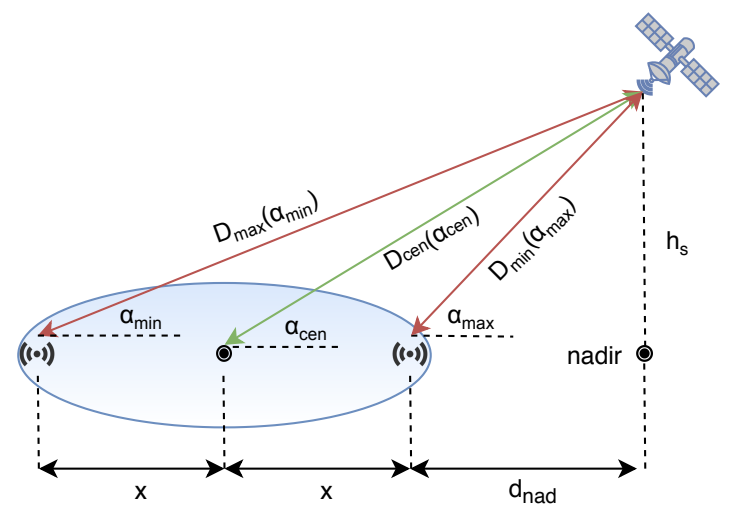

Fig. 7. System Geometry

on the NPRACH format used for the preamble transmission. There exist three NPRACH formats in NB-IoT. The NPRACH Format 0 and 1 were introduced in Release 13 , and use a CP length of $66.7 \mu \mathrm{s}$ and $266.67 \mu \mathrm{s}$ respectively, with $3.75 \mathrm{kHz}$ subcarrier spacing (SCS). Whereas, the NPRACH Format 2 was introduced in Release 15, and utilizes a CP duration of $800 \mu s$ with $1.25 \mathrm{kHz}$ SCS. By plugging in the CP length values in Equation (4), we can derive the three possible cell radiuses for the terrestrial case, as reported in the standard [14], corresponding to $10 \mathrm{~km}, 40 \mathrm{~km}$ and $120 \mathrm{~km}$.

The derivation of the cell size for an NB-IoT NTN will be different, due to the particular geometry with respect to the terrestrial case. To compute the maximum cell size, we consider the worst-case scenario in which two UEs are located in the edges of the cell on the major axis direction (please refer to Fig. 7). This corresponds to the maximum differential RTD experienced in a specific cell, and can be computed from the differential slant range among those two UEs, as in [15]:

$$
\begin{aligned}
& \Delta D=D_{\max }\left(\alpha_{\min }\right)-D_{\min }\left(\alpha_{\max }\right) \\
& =\left(\sqrt{R_{E}^{2} \sin \left(\alpha_{\min }\right)^{2}+h_{s}^{2}+2 R_{E} h_{s}}-R_{E} \cdot \sin \left(\alpha_{\min }\right)\right) \\
& -\left(\sqrt{R_{E}^{2} \sin \left(\alpha_{\max }\right)^{2}+h_{s}^{2}+2 R_{E} h_{s}}-R_{E} \cdot \sin \left(\alpha_{\max }\right)\right)
\end{aligned}
$$

Notably, the maximum slant range corresponds to the minimum elevation angle, and vice versa. To make sure that the differential RTD does not exceed the allowed limit, we use as a bound the cell radius calculated in Equation (4) for the terrestrial case:

$$
\Delta D \leq R_{\text {cell }}^{(R A)}
$$

For a certain cell with a minimum elevation angle $\alpha_{m i n}$, the respective slant range $D_{\max }$ can be calculated from Equation (3). To compute the minimum allowed slant range $D_{\min }$ and the corresponding elevation angle $\alpha_{\max }$, we need to solve the following equation: 


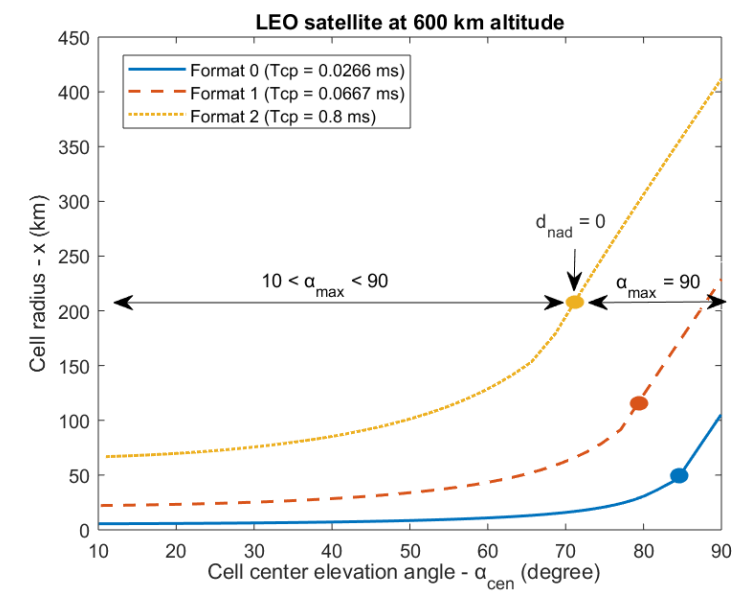

Fig. 8. Cell radius vs Cell center elevation angle

$$
\begin{aligned}
D_{\min }\left(\alpha_{\max }\right) & =\min _{\alpha} D(\alpha) \\
& \text { such that: } \\
& D_{\max }\left(\alpha_{\min }\right)-D(\alpha) \leq R_{\text {cell }}^{(R A)} \\
& \alpha \in\left[\begin{array}{ll}
\alpha_{\min } & 90^{\circ}
\end{array}\right]
\end{aligned}
$$

It can be easily solved through numerical simulations. At this point, after having obtained $\alpha_{\max }$ and its respective slant range, we can calculate the radius of the cell by the low of cosines in a triangle.

$$
x=\frac{\sqrt{D_{\max }^{2}+D_{\min }^{2}+2 D_{\max } D_{\min } \cos \left(\alpha_{\max }+\alpha_{\min }\right)}}{2}
$$

It is obvious that the cell radius $x$ has a dependency on the minimum elevation angle of that particular cell. Fig. 8 depicts the results, after performing numerical simulations in Matlab by using Equation (3)(7) and (8), for distinct values of $\alpha_{\min }$ and three existing NPRACH configurations. For simplicity, we have plotted the results as a function of the elevation angle at the center of the beam $\alpha_{c e n}$, belonging to a particular $\alpha_{\min }$ and cell radius $x$ (we have skipped the derivation here). The emphasized dots in the graph represent the points where the $\alpha_{\max }$ reaches 90 degrees, hence the minimum slant range will coincide with the satellite altitude $D_{\min }=h_{s}$. From this point onward, the closest user to the satellite is always the one placed at the nadir, not the one at the edge of the coverage area. The cell radius increases very fast (it doubles) with the increase of the cell center elevation angle, while the maximum is reached when the center of the beam is at the satellite nadir. Please note that we have not shown the results for a GEO satellite because the behaviour is the same. Obviously, the higher the orbit the larger the cell radius $x$ would be. To interpret the impact of these results on the RA procedure, we divide the analysis for the two cell types.

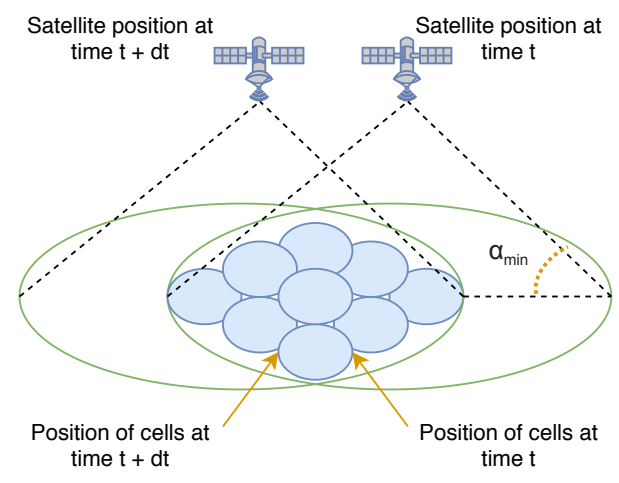

Fig. 9. Earth-fixed cells example

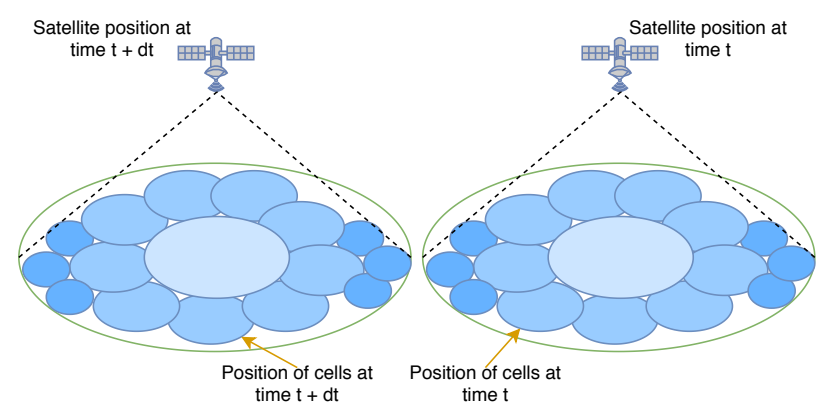

Fig. 10. Earth-moving cells example

1) Earth-fixed cell: In this case, the related parameters of the cell with the satellite will change over time. Therefore, it is important to take into account the worst-case scenario when fixing the cell on Earth. Please note that the worst-case scenario of maximum RTD, resulting in the lowest coverage radius, occurs when the satellite is close to the horizon. For a minimum elevation angle of 10 degrees, the Earth-fixed cell will have a radius of approximately $6 \mathrm{~km}, 22 \mathrm{~km}$ and 70 $\mathrm{km}$ for NPRACH Format 0,1 and 2 respectively. An example scenario is shown in Fig. 9 for a fixed NPRACH configuration. A rough estimation, by considering only the surfaces of the cell and the satellite coverage (minimum elevation angle of 10 degrees) for the best-case scenario (NPRACH Format 2), results in around 730 cells that could potentially be covered by one LEO satellite simultaneously.

2) Earth-moving cell: In such a scenario, the relation of a certain cell with the satellite does not change over time. Consequently, given the elevation angle of the center of the cell, we can derive its respective radius. Notably, the closer the cell is to the satellite nadir, the bigger its radius would be. Again, an illustration of this scenario under a specific NPRACH configuration is shown in Fig. 10. Compared to the Earth-fixed cell, the number of potential cells will be lower, approximately around 450 for the best-case scenario (NPRACH Format 2).

If only one NB-IoT carrier is available for all the cells under the coverage of the satellite, two UEs in different cells trying to access the network at the same time, would fail to have 


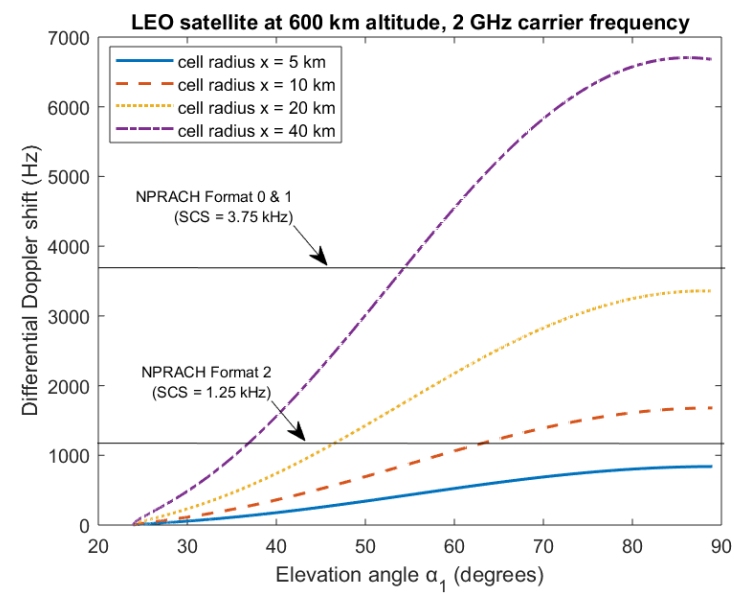

Fig. 11. Differential Doppler shift vs Elevation angle

a successful RA procedure. A straightforward solution would be to assign to each cell a separate NB-IoT carrier. Multicarrier operation is supported by the standard and introduced in Release 15 [14]. However, this would lead to significant waste of spectrum, taking into account the large amount of cells, the low demand (especially in remote areas where the satellite would play a role), and the sporadic nature of the traffic coming from the UEs on ground. Alternative solutions should be proposed, with the aim to increase the cell size according to the UE demand, while minimizing the number of required NB-IoT carriers.

\section{Differential Doppler shift}

Doppler effects are quite a well-known problem in a LEO satellite-based communication system. While in a terrestrial network the Doppler shift is caused by the movement of the UEs, in a satellite communication system, an added factor is the high-speed movement of the satellite. A part of this Doppler shift will be common for all the users inside the same cell (for example the one in the center of the cell), and solutions exist in the literature on how to efficiently pre/post compensate it at the Gateway [16]-[18]. In addition, since the UEs on ground will have different locations, there will be a differential Doppler shift among them causing an overlap of their preambles. Obviously, the higher the overlap, the lower the probability of a successful RA procedure would be.

In our previous work [7], we propose a resource allocation strategy in order to limit the differential Doppler shift up to a level supported by the standard. Basically, the main concept behind is to schedule at the same radio frames only UEs which do not violate the differential Doppler limit. While this solution is well-suitable for message 3 transmission of the RA procedure (and all subsequent transmissions), it is not applicable to message 1 . This is due to the fact that in message 1 the transmission is not controlled by the BS. The UEs compete for the same NPRACH channel and they randomly select the resources to use, subject to some pre-configured preamble formats.

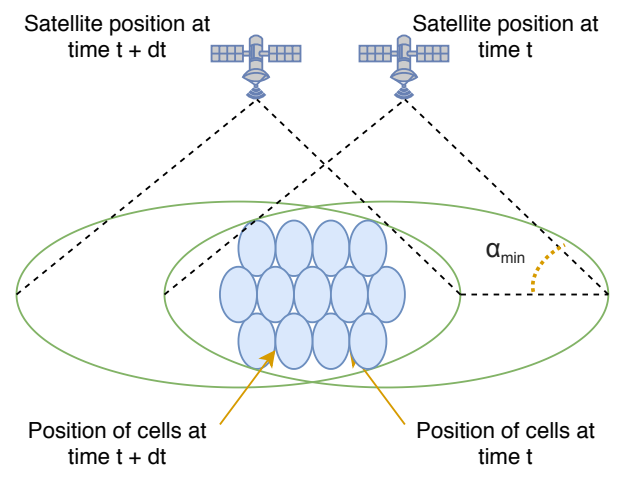

Fig. 12. Earth-fixed cells example

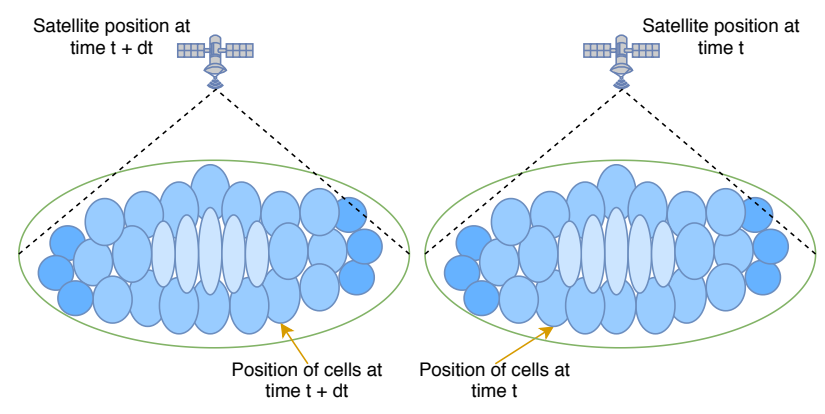

Fig. 13. Earth-moving cells example

As demonstrated in [7], the main contributor towards the differential Doppler shift is the change of position along $\mathrm{x}$ axes (the direction of the satellite movement), whereas the $y$ axes (perpendicular to the direction of the satellite movement) differential Doppler is negligible. To calculate the maximum differential Doppler shift given the cell radius $x$, we use the following formula as derived in [7]:

$$
\Delta f_{d}^{\max }=\left|\frac{f_{c} w_{s} R_{E}}{c} \cdot\left(\cos \left(\alpha_{1}\right)-\cos \left(\alpha_{2}\right)\right)\right|
$$

where $w_{s}$ is the angular velocity of the satellite, which can be easily derived by knowing the satellite altitude, $f_{c}$ is the NB-IoT carrier frequency, $\alpha_{1}$ and $\alpha_{2}$ are the elevation angles of the UEs experiencing the maximum differential Doppler shift (placed at a maximum distance of $2 x$ along x-axes). As we can see from the plotted results in Fig. 11, the maximum differential Doppler shift increases proportionally with the increase of the elevation angle and cell radius, for a fixed carrier frequency $f_{c}=2 \mathrm{GHz}$. For example, having a cell radius higher than $20 \mathrm{~km}$, means that the preambles may totally overlap for NPRACH Format 0 and 1 at high elevation angles.

The limit that the standard can support is not known for the RA procedure. However, we might need to keep it as low as possible in order to minimize the overlap among preambles, hence increasing the chances of a successful RA procedure. The only way to decrease the differential Doppler shift, without modifying the existing standard, is to tighten the 
cells. Of course, to what extent, will depend on the probability of detection target of the RA procedure. Since the main contributor towards the differential Doppler shift is the change of position along $\mathrm{X}$-axes, we obtain smaller ellipsoid cells as illustrated in Fig. 12 and Fig. 13. Again, for the Earth-fixed cells we have to take into account the worst-case scenario, whereas for the Earth-moving cell, depending on the elevation angle, the cells will have different $\mathrm{x}$-axes sizes.

Compared to the analysis done in Section IV-B, the number of created cells which take into account both, the differential RTD and differential Doppler shift, would be considerably higher. As a consequence, the problem we already stated in Section IV-B would be reinforced.

\section{Frequent Outdate of the TA value}

As we previously emphasized, one of the reasons for the $\mathrm{RA}$ procedure is to ensure the time synchronization among UEs, through the TA value reported in message 2 . It is evident that in our NB-IoT NTN scenarios with LEO orbit, due to the high-speed movement of the satellite, the relation of users inside a cell (either Earth-fixed or Earth-moving) will change quickly over time. This will cause the UEs to loose the timesynchronization, and repeat the RA procedure in order to get an updated TA value. A frequent RA procedure will lead to a RA congestion, and directly impact the overall throughput and the capacity of the NB-IoT network. Solutions should be found to keep the caused RA congestion under control.

\section{PROPOSED SOLUTIONS AND RESEARCH DIRECTIONS}

In this section, we will outline some solutions and research directions, in order to overcome the challenges investigated in Section IV. Part of the proposals have already been discussed in the context of 5G NTN systems [6], [16], [17], and their feasibility for the NB-IoT NTN case will be analyzed.

\section{A. New NPRACH configurations with extended RAR window sizes and CP length}

The new RAR window sizes should be able to cope with the increased RTD of communication over the satellite channel. For the worst-case scenario of a GEO satellite with transparent payload, the RAR window size should be greater than $540 \mathrm{~ms}$. This value is much higher than the maximum window size of $41 \mathrm{~ms}$ in case of a terrestrial NB-IoT network. Of course, other values should be included for all the possible scenarios and orbit altitudes.

To increase the coverage of the cells, new preambles with longer CP lengths are needed. This will allow the BS to have more degrees of freedom in selecting the size of the cells, and adapt them subject to the required traffic and number of UEs at a specific region on Earth.

\section{B. Extended downlink control information (DCI) field}

The additional NPRACH configurations, bring up the need of extra information to be reported to the UEs by the BS. In a terrestrial network, to select an appropriate NPRACH configuration, the UEs rely on the estimate of the received signal power by the BS. This is not enough in an NTN scenario because the estimated received signal power cannot indicate the orbit/type of the satellite, under which the UEs are covered with service.

Therefore, new fields should be included in the DCI containing information regarding the satellite altitude and payload type. This would greatly help the UEs to take proper decisions regarding the NPRACH configuration (having various RAR window sizes or $\mathrm{CP}$ lengths as previously explained). For example, for the six scenario options identified in our paper, at least 3 extra bits are needed in the DCI field, to indicate to the UEs under which scenario are being covered.

In addition, other fields can be included in the DCI to continuously update the UEs with the new TA value. Obviously, the estimation of the TA has to be done at the BS, by using the ToA of distinct signals coming from many UEs. By doing so, the UEs in an RRC connected mode, will be able to keep the time-synchronization and avoid multiple RA procedures.

\section{Disabled HARQ protocol}

Disabling the HARQ protocol would significantly reduce the message exchange between the UEs and the BS. This is highly desirable for the RA procedure over a satellite, first because it would enable a faster uplink data transmission, and second more UEs would be able to access the network.

However, it is worth highlighting here that to keep the same link reliability as in the case of HARQ operation, the block error rate (BLER) target should be properly adjusted.

\section{GNSS solutions for differential RTD/Doppler estimation}

This technique is also proposed by the authors in [6], [16], [17] for the 5G NTN. It is based on the capability of the UEs to estimate their location and the position of the satellite. An ideal estimation, would lead to a perfect pre-compensation of the differential Doppler shift and RTD at the user side. Thereby, the frames from various UEs will be aligned both, in time and frequency, regardless of the cell size. In such a case, even a cell as large as the satellite coverage, would be able to operate without facing the time-frequency misalignment problem.

The applicability of GNSS solutions for NB-IoT NTN is questionable. Although in the next NB-IoT releases, the UEs will have the GNSS positioning capability, frequent estimation of the differential Doppler/RTD to be compensated (typical for NB-IoT over LEO) means extra processing, diminishing the device battery lifetime. A straightforward solution to this, as also used in other practical systems, would be to periodically disable the GNSS and use the latest position information for differential Doppler/RTD estimation. While this decreases the required processing, it impacts the accuracy of the estimation.

\section{E. Group-based RA}

An alternative solution to the one proposed in Section V-D is the group-based RA. The main concept behind is to use the same NB-IoT carrier for more than one cell, but at different times. This means that only the UEs inside a certain cell are allowed to start the RA procedure for a period of time. For 
another period, the BS will allow users of another cell to access the same NB-IoT carrier. Following this approach, it is also possible to adapt to different demands coming from various cells. The higher the demand, the longer the NB-IoT carrier will be available for the UEs to perform the RA procedure. If only one NB-IoT carrier is not enough to support all the cells, secondary ones may be added. No complexity is added at the user side, but only at the BS, being the responsible entity for resource allocation.

Another advantage of this access mechanism is that it reduces the RA congestion, which is also a well-known problem in a terrestrial network. Since fewer devices will be granted to access the network at the same time, the RA detection probability can be increased.

The only drawback of this approach is that the access will be always initiated by the BS and not by the UEs. After achieving downlink synchronization, the device needs to first check whether its access class (different classes for different cells) is allowed to access the network. In case the device is barred (belongs to another class), it should back off and then reattempt access at a later point in time. The information regarding the UE class is reported in the system information block (SIB) in the downlink transmission, as specified in the standard.

\section{F. Early data transmission (EDT)}

As previously explained, in an Earth-fixed cell scenario, the movement of the satellite causes the related parameters among users and the satellite to constantly change over time. On the other hand, for an Earth-moving cell scenario, the UEs will be constantly moving in and out a certain cell. Such a dynamic system brings up the need for transmitting the uplink data as soon as possible in the RA procedure, thus avoiding extra message exchange between the BS and the UEs. In fact, the NB-IoT Release 15 has already introduced the concept of early data transmission in message 3 of the RA procedure. This technique would be very suitable for an NB-IoT NTN. The difference is that while in a terrestrial NB-IoT the EDT is projected to be used in certain scenarios (reduce the delay or increase the battery life), for an NB-IoT NTN this would be the default solution for data transmission.

The disadvantage of this approach is that the BS will be unable to assign the resources for uplink data transmission according to the UE demand, because the data volume that the UEs has to send to the BS is reported in message 3.

\section{CONCLUSIONS}

In this paper, we considered an NB-IoT system over satellite and identified six scenario options, based on the satellite orbit, payload and cell type. By referring to the latest 3GPP specifications, we addressed the impact of the typical channel impairments on the NB-IoT random access procedure. We proposed new NPRACH configurations with extended RAR window sizes and $\mathrm{CP}$ length, which take into account the increased RTD and differential RTD in the satellite channel. The additional NPRACH configurations, bring the need for extra information to be reported to the UEs by the BS, which can be included in the DCI. To increase the coverage of the cells, opposed to GNSS solutions, group-based RA can be a less complex one, which not only is able to adapt to different demands coming from various cells, but also helps to reduce the RA congestion. Last but not least, due to the dynamicity of our NB-IoT NTN system, we propose to deactivate the HARQ protocol and use EDT, in order to limit as much as possible the data exchange between the UEs and the BS.

\section{REFERENCES}

[1] 3GPP TR 38.811, “Technical Specification Group Radio Access Network; Study on New Radio (NR) to support non-terrestrial networks (Release 15) ," Tech. Rep., September 2019.

[2] 3GPP TR 38.821, "Technical Specification Group Radio Access Network; Solutions for NR to support non-terrestrial networks (NTN) (Release 16) ," Tech. Rep., December 2019.

[3] 3GPP RP-193235, "Study on NB-Io/eMTC support for Non-Terrestrial Network ," Tech. Rep., December 2019.

[4] S. Cioni, R. De Gaudenzi, O. Del Rio Herrero, and N. Girault, "On the satellite role in the era of $5 \mathrm{~g}$ massive machine type communications," IEEE Network, vol. 32, no. 5, pp. 54-61, Sep. 2018.

[5] N. Alagha, "Satellite air interface evolutions in the $5 \mathrm{~g}$ and iot era," SIGMETRICS Perform. Eval. Rev., vol. 46, no. 3, p. 93-95, Jan. 2019. [Online]. Available: https://doi.org/10.1145/3308897.3308941

[6] A. Guidotti et al., "Architectures and key technical challenges for $5 \mathrm{~g}$ systems incorporating satellites," IEEE Transactions on Vehicular Technology, vol. 68, no. 3, pp. 2624-2639, March 2019.

[7] O. Kodheli, S. Andrenacci, N. Maturo, S. Chatzinotas, and F. Zimmer, "Resource allocation approach for differential doppler reduction in nbiot over leo satellite," in 2018 9th Advanced Satellite Multimedia Systems Conference and the 15th Signal Processing for Space Communications Workshop (ASMS/SPSC), Sep. 2018, pp. 1-8.

[8] J. Doré and V. Berg, "Turbo-fsk: A $5 \mathrm{~g}$ nb-iot evolution for leo satellite networks," in 2018 IEEE Global Conference on Signal and Information Processing (GlobalSIP), Nov 2018, pp. 1040-1044.

[9] S. Cluzel et al., "3gpp nb-iot coverage extension using leo satellites," in 2018 IEEE 87th Vehicular Technology Conference (VTC Spring), June 2018, pp. 1-5.

[10] H. Saarnisaari, A. O. Laiyemo, and C. H. M. de Lima, "Random access process analysis of $5 \mathrm{~g}$ new radio based satellite links," in 2019 IEEE 2nd 5G World Forum (5GWF), Sep. 2019, pp. 654-658.

[11] 3GPP RP-180581, "Interim conclusions on IoT for Rel-16," Tech. Rep. March 2018.

[12] Olof Liberg Marten Sundberg Eric Wang Johan Bergman Joachim Sachs Gustav Wikstrom, Cellular Internet of Things : From Massive Deployments to Critical $5 G$ Applications, 2nd Edition. Elsevier Science \& Technology, 2020.

[13] TS 36.321, "Technical Specification Group Radio Access Network; Evolved Universal Terrestrial Radio Access (E-UTRA); Medium Access Control (MAC) protocol specification (Release 15) ," Tech. Rep., December 2019.

[14] 3GPP TS 36.213, "LTE; Evolved Universal Terrestrial Radio Access (EUTRA); Physical layer procedures (version 15.7.0 Release 15) ," Tech. Rep., October 2019.

[15] A. Guidotti, "Beam size design for new radio satellite communications systems," IEEE Transactions on Vehicular Technology, vol. 68, no. 11 pp. 11379-11383, 2019.

[16] A. Guidotti et al., "Satellite-enabled lte systems in leo constellations," in 2017 IEEE International Conference on Communications Workshops (ICC Workshops), May 2017, pp. 876-881.

[17] O. Kodheli, A. Guidotti, and A. Vanelli-Coralli, "Integration of satellites in 5g through leo constellations," in GLOBECOM 2017 - 2017 IEEE Global Communications Conference, Dec 2017, pp. 1-6.

[18] Jionghui Li et al., "Fast tracking doppler compensation for ofdmbased leo satellite data transmission," in 2016 2nd IEEE International Conference on Computer and Communications (ICCC), Oct 2016, pp. 1814-1817. 